\title{
Peptide Drug Thymalin Regulates Immune Status in Severe COVID-19 Older Patients
}

\author{
B. Kuznik ${ }^{a, ~ *}$, V. Khavinson ${ }^{b, c}$, K. Shapovalov ${ }^{a}$, N. Linkova ${ }^{b}$, S. Lukyanov ${ }^{a}$, Yu. Smolyakov ${ }^{a}$, \\ P. Tereshkov ${ }^{a}$, Yu. Shapovalov ${ }^{a}$, V. Konnov ${ }^{a}$, and N. Tsybikov ${ }^{a}$ \\ ${ }^{a}$ Department of the Normal Physiology, Chita State Medical Academy, Chita, 672000 Russia \\ ${ }^{b}$ Department of Biogerontology, Saint Petersburg Institute of Bioregulation and Gerontology, St. Petersburg, 197110 Russia \\ ${ }^{c}$ The Group of Peptide Regulation of Aging, Pavlov Institute of Physiology, \\ Russian Academy of Sciences, St. Petersburg, 199034 Russia \\ *e-mail:miayy@yandex.ru \\ Received May 24, 2021; revised July 16, 2021; accepted July 22, 2021
}

\begin{abstract}
Peptide drug Thymalin, isolated from the calve thymus, is successfully used for the treatment of various immunopathologies, including those in older age groups. The molecular mechanism of the Thymalin immunoprotective action is due to the effects of the short peptides KE, EW, EDP in its composition. These short peptides can specifically bind to double-stranded DNA and/or histone proteins and regulate gene expression, synthesis of immune system proteins, activity of gerontogenes, and stimulate stem cell differentiation. Regulation of immunogenesis is a key factor preventing the development of the "cytokine storm" that develops in severe COVID-19. The purpose of this work is to study the effectiveness of Thymalin in severe COVID-19 in older patients. Patients administered with Thymalin against the background of a standard therapy $(n=36)$ manifested a more rapid clinical improvement, higher proportions of recovery from lymphopenia, faster normalization of the concentration of C-reactive protein, D-dimer, the number of lymphocytes and NK-cells in the blood, compared to patients who received a standard therapy only $(n=44)$. Thymalin halved hospital mortality in older patients with severe COVID-19. The results obtained showed the effectiveness of Thymalin administration in the complex therapy of patients with severe COVID-19.
\end{abstract}

Keywords: Thymalin, peptides, immunity, severe COVID-19, older people

DOI: $10.1134 /$ S2079057021040068

\section{INTRODUCTION}

Thymalin (registration number LS-000267 dated February 26, 2010, Ministry of Health of the Russian Federation) is a medicinal drug based on a polypeptide complex isolated from the calve thymus. Thymalin is effective in case of various infectious diseases (hepatitis $\mathrm{A}$ and $\mathrm{B}$, erysipelas, meningococcal infection, typhoid fever), acute respiratory diseases, pneumonia of various origins, cavernous pulmonary tuberculosis $[2,14,15]$. Administration of Thymalin reduced the incidence of acute respiratory infections in senior patients 2.0-2.4-fold [32].

Thymalin reduced mortality in senior patients over a 6-year observation period [12]. Thymalin is accumulated in epithelial cells of the human respiratory tract during early embryogenesis [20]. Thymalin prevents the "cytokine storm," which develops during COVID19 , reducing the concentration of pro-inflammatory cytokines IL-l $\alpha$, IL-6, IL-8, TNF- $\alpha$ in the blood [18]. Thymalin reduces the intensity of intravascular blood coagulation in the development of hypercoagulability syndrome $[14,15]$, which is known as a com- mon cause of complications in patients with severe COVID-19.

A case of a successful Thymalin administration in an older patient with a severe form of COVID-19, developed on the background of chronic diseases (type II diabetes mellitus, stage III hypertension, ischemic heart disease), was reported [18]. Combination of Thymalin and comprehensive treatment allowed to arrest the "cytokine storm", increase the concentration of blood lymphocytes 2-fold, eosinophils-7fold, reduce the concentration of $\mathrm{C}$-reactive protein, IL-6, D-dimer by 8, 2.5 and 7.2 times, respectively. It is noteworthy that initially the patient received only standard therapy, in the course of which his condition worsened.

In a study on human hematopoietic blood stem cells (HSC), Thymalin decreased the expression of CD44 on HSC 2.8-fold [19]. CD44 is a ligand for Eand L-selectin, which maintains the HSC subpopulation. CD44 is involved in the activation of proliferation, HSC survivability enhancement and modulation of cytokine action [8, 26]. Thymalin reduced the 
expression of CD117 on HSC 2.2-fold [19]. CD117 is a receptor for stem cell growth factor; it stimulates their proliferation and early stages of differentiation [27]. CD117 expression decreases during differentiation of immune cells [8]. Thymalin reduced the expression of CD28 on HSC 6.9-fold [19]. CD28 is expressed on $\mathrm{CD} 4^{+}, \mathrm{CD} 8^{+}$lymphocytes and is involved in the activation of mature T cells. CD28 expression increases in antigen-presenting cells upon activation of toll-like receptors and plays an essential part in the activation of immunity in viral diseases. Patients with mild forms of COVID-19 manifest an increase in the number of $\mathrm{CD}^{+}, \mathrm{CD}^{+}, \mathrm{CD} 28^{+}$lymphocytes, which indicates the activation of the immune system functions. In severe cases of coronavirus infection, the number of $\mathrm{T}$-helpers and cytotoxic lymphocytes decreases alongside a simultaneous decline in the expression of CD28 on their surface [36]. Thus, Thymalin action on the differentiation of human HSCs, including the increase in the number of $\mathrm{CD} 28^{+}$cells in the blood, may be one of the mechanisms, activating the immune response in patients with COVID-19.

According to the molecular mechanism of action, Thymalin belongs to the group of drugs that epigenetically regulate gene expression and synthesis of immunogenesis proteins. Thymalin normalizes differentiation, proliferation and reduces apoptosis of lymphoid cells. Immunoprotective and geroprotective properties of Thymalin are due to the effects of short peptides EW, KE, EDP in its composition [3, 13, 17, 35]. Peptides EW, KE, EDP, which comprise the medicinal drug Thymalin, are able to penetrate into cell nucleus and nucleolus and complementary bind to certain DNA sequences and/or histone proteins [6, 7, 16, 21]. This binding results in a change in gene expression and synthesis of heat shock proteins, cytokines, fibrinolysis system, gerontogenes and proteins, involved in the differentiation, proliferation and apoptosis of immune cells. Normalization of the synthesis of proteins, which activate the immune and antioxidant systems' functions, regeneration processes and hemostasis, manifests itself in the restoration of the functions of the body's regulatory systems and explains the high efficiency of Thymalin in the treatment of a number of diseases.

Thus, Thymalin, an epigenetic regulator of gene expression and immunogenesis protein synthesis, can be considered a potentially effective agent for the complex therapy in patients with severe COVID-19. The purpose of this work is to study the effectiveness of Thymalin in severe COVID-19 in older patients.

\section{EXPERIMENTAL}

This prospective, randomized single-blind controlled clinical trial was performed at the Chita State Medical Academy Hospital in Chita, Russia. Patients meeting the following criteria were enrolled in the study: over 18 years of age, regardless of gender; positive respiratory samples for SARS-CoV-2 nucleic acid (samples were tested for SARS-CoV-2 virus genetic material using reverse transcription polymerase chain reaction (RT-PCR); COVID-19 was diagnosed according to the diagnostic criteria of "the latest Clinical guidelines for the novel coronavirus" issued by the World Health Organization (WHO) on January 28, 2020 [11].

The study included COVID-19 patients meeting the following criteria for critical disease: the patients had pneumonia with lung involvement characteristic of COVID-19 with at least 50\% lung tissue damage (CT3 or CT-4 according to the Clinical Guidelines); the patients required supplemental oxygen to maintain oxygen saturation $\left(\mathrm{SpO} \mathrm{O}_{2}\right)$ less than 93\%. The study excluded patients with severe immunosuppression due to HIV infection, long-term use of corticosteroids (>10 mg prednisolone/day) and/or cytostatics, oncological diseases, patients treated with IL-6 receptor inhibitors and JAK-kinase inhibitors.

The enrolled patients were randomly allocated into 2 groups by an independent statistician. The process of randomization was masked to all treating physicians. Patient unique identification number and treatment allocation codes were provided by a clinical research associate. The enrolled patients were randomly divided in 2 groups: control group $(n=44)$, which received standard COVID-19 treatment and placebo ( $2 \mathrm{~mL} \mathrm{NaCl} 0.9 \%$ saline, intramuscular injections) daily for 10 days; and investigation group $(n=36)$, which received standard COVID-19 treatment and Thymalin by $10 \mathrm{mg} / 2 \mathrm{~mL} 0.9 \% \mathrm{NaCl}$ in the form of intramuscular injections daily for 10 days. Standard COVID-19 treatment was based on the seventh edition of the Russian Ministry of Health's guidelines for the prevention, diagnosis and treatment of the new coronavirus infection COVID-19 (https://static-0.rosminzdrav.ru/system/attachments/attaches/000/050/ 584/original/03062020_\%D0\%9CR_COVID-19_v7.pdf). This treatment included antiviral therapy (monotherapy with hydroxychloroquine or a combination of drugs with antiretroviral activity-lopinavir + ritonavir), antibacterial agents (with clinical symptoms of bacterial infection; parenteral drugs: beta-lactam drug/beta-lactamase inhibitor + macrolide or respiratory fluoroquinolone), anticoagulant therapy (enoxaparin sodium $1 \mathrm{mg} / \mathrm{kg}$ bid subcutaneously), supplemental oxygen, noninvasive and invasive ventilation, corticosteroid, vasopressor support, renal-replacement therapy, and extracorporeal membrane oxygenation.

The patients underwent non-contrast-enhanced chest computer tomography (CT) right after they were divided into groups and on the 14th day. If the condition deteriorated, an additional chest CT was performed. Disease progression was determined by comparing the number, volume, and density of lung 
lesions on two chest CT scans. Fusions of previous lung lesions, new lesions, and increased lung density were considered as progression [11].

Peripheral blood was taken immediately after the patients were divided into groups and on the 14th day to determine the complete blood cell count, serum biochemical parameters, high-sensitivity C-reactive protein (CRP), IL-6, D-dimer, fibrinogen, and immunophenotyping of lymphocytes by flow cytometry. Complete blood count analysis was immediately after the patients were divided into groups and on the 14th day on a Sysmex XN-1000 hematological analyzer (Sysmex Corporation, Chuo-ku, Kobe 6510073, Japan). Serum IL-6 levels were determined by enzyme-linked immunosorbent assay (ELISA) method using a commercial kit for the determination of IL-6 (JSC "Vector-Best," Russia). The results were recorded using the program for calculating concentrations by multipoint calibration on an Expert 96 microplate photometer (ASYS HITECH, Austria) at a wavelength of $450 \mathrm{~nm}$. Peripheral blood lymphocyte subset counts were quantified by flow-cytometric immunophenotyping using standardized protocols on a CytoFLEX LX flow cytometer ("Beckman Coulter," United States) equipped with four diode lasers 355, 405, 488, and $561 \mathrm{~nm}$. Monoclonal antibodies to determine T, B, NK, and NK cell subsets were purchased from Beckman Coulter. The following antibodies were used: TCR PAN $\alpha / \beta-P E$ (clone IP26A, cat. no. B49177), CD19-ECD (clone J3-119, cat. no. IM2708U), CD14-PC5 (clone RMO52, cat. no. IM2640U), CD56-PC7 (clone N901 (NKH-1), cat. no. A51078), CD16-Pacific Blue (clone 3G8, cat. no. A82792), CD45-Krome Orange (clone J.33, cat. no. A96416); antibodies manufactured by Becton Dickinson, USA: CD4-BUV395 (clone RPA-T4, cat. no. 564724), CD8-BUV496 (clone RPA-T8, cat. no. 612942), CD3-BUV661 (clone UCHT1, cat. no. 612964); antibody from Biolegend, USA: HLA-DRBrilliant Violet $785^{\mathrm{TM}}$ (clone L243, cat. no. 307642). Cytofluorometric data were processed using CytExpert software v.2.0 and Kaluza ${ }^{\mathrm{TM}}$ v.2.1.1 ("Beckman Coulter," United States).

The primary efficacy endpoints were: percentage of clinical improvement, time to clinical improvement, defined as the time from the division of patients into groups to a 2-point improvement on a 7-category ordinal scale (recommended by the World Health Organization: Coronavirus disease (COVID-2019) R\&D. Geneva: World Health Organization) [5, 24] and the improvement rate of the follow-up CT scans on the 14th day. The secondary endpoints were: inhospital mortality, the time from the division of patients into groups to death, lymphocyte recovery and the time to lymphocyte recovery, COVID-19 virus clearance time among the survivors.

Statistical analysis was performed using the R language version 3.6.2. Continuous variables were expressed as $M \pm S D$ (Mean \pm Standard deviation). To compare the parameters that were not distributed normally, the Wilcoxon tests were used. The Wilcoxon signed-rank test with Hommel correction was used for multiple pairwise comparisons of the patient data on different days of treatment. To compare several independent groups, the Wilcoxon rank-sum test with Hommel correction was used. $p$ value $<0.05$ was considered statistically significant.

\section{RESULTS AND DISCUSSION}

Initially there was no significant difference between the two groups in demographic characteristics, clinical severity, comorbidity, respiratory support modality, concomitant therapy (Table 1). Administration of Thymalin resulted in a statistically significant increase in the number of blood leukocytes by $25 \%$, while standard therapy revealed no such results. Thymalin contributed to a reliable increase in the number of blood lymphocytes by $92 \%$ compared to the indicator before treatment. At the same time, initial number of blood lymphocytes in the control group was higher than in the group subsequently administered with Thymalin. Standard treatment had no significant effect on the number of blood lymphocytes in patients with coronavirus infection. The number of blood monocytes after standard treatment and Thymalin administration increased by 42 and 55\%, respectively, compared to the initial values in the studied groups. Thymalin and standard therapy increased the concentration of blood eosinophils 4.6- and 3.1-fold, respectively, compared to the baseline values in each group. Administration of Thymalin increased the number of T- and B-lymphocytes, NK-cells in the blood by 2.2 , 2 and 2.4 times compared to the initial value, while standard therapy did not affect these indicators significantly. Thymalin increased the number of $\mathrm{CD} 4^{+}$, $\mathrm{CD}^{+}, \mathrm{CD}^{+}{ }^{+} \mathrm{HLA}^{-} \mathrm{DR}^{+}$cells in the blood by $2.2,2.2$ and 3.4 times compared to the base values, while standard treatment had no such effect.

After standard treatment and Thymalin administration the number of blood platelets grew up by 35 and $42 \%$, respectively, compared to the initial values in the studied groups. Moreover, the number of blood platelets in patients with COVID-19 was significantly higher after the use of Thymalin, compared to standard therapy. There was a statistically significant increase in the platelet/lymphocyte ratio after Thymalin administration and standard therapy by 27 and $21 \%$, respectively. Thymalin reduced the amount of fibrinogen in patients with coronavirus infection by $30 \%$ compared to the same indicator before treatment, while standard therapy had no such effect. The concentration of C-reactive protein in the blood decreased significantly after the use of Thymalin and standard treatment by 3.3 and 2.2 times, respectively. Thymalin reduced the concentration of IL-6, D-dimer and lactate dehydrogenase in the blood by $6.5,1.5$ and 1.9 
Table 1. Demographic and clinical characteristics of the patients

\begin{tabular}{|c|c|c|c|}
\hline Characteristics & Control group & Investigation group (Thymalin) & $p$ \\
\hline Age, years $(M \pm S D)$ & $61.95 \pm 13.43$ & $61.97 \pm 9.76$ & 0.92 \\
\hline Sex (female), $n, \%$ & $21(47.7)$ & $16(44.4)$ & 0.77 \\
\hline Smoking history, $n, \%$ & $14(31.8)$ & $11(30.55)$ & 0.97 \\
\hline Body mass index, $M \pm S D$ & $29.83 \pm 4.08$ & $30.03 \pm 4.36$ & 0.89 \\
\hline \multicolumn{4}{|c|}{ Severity } \\
\hline ICU patients, $n, \%$ & $34(77.2)$ & $28(77.7)$ & 0.96 \\
\hline Respiratory support (NiV/IMV), $n, \%$ & $25(56.8)$ & $20(55.5)$ & 0.91 \\
\hline $\begin{array}{l}\text { CT visual quantitative evaluation of acute lung lesions } \\
\text { score III-IV ( }>50 \% \text { lung involvement), } n, \%\end{array}$ & $37(84.1)$ & $30(83.3)$ & 0.93 \\
\hline \multicolumn{4}{|c|}{ Comorbidity } \\
\hline Hypertension, $n, \%$ & $29(65.9)$ & $23(63.8)$ & 0.85 \\
\hline Diabetes, $n, \%$ & $10(22.7)$ & $8(22.2)$ & 0.96 \\
\hline Coronary artery heart disease, $n, \%$ & $27(61.3)$ & $20(55.5)$ & 0.61 \\
\hline Obesity, $n, \%$ & $22(50)$ & $17(47.2)$ & 0.83 \\
\hline Days from illness onset to randomization, $M \pm S D$ & $8.55 \pm 1.65$ & $8.88 \pm 1.46$ & 0.81 \\
\hline \multicolumn{4}{|c|}{ Treatments during study period } \\
\hline Noninvasive ventilation, $n, \%$ & $19(43.18)$ & $20(55.5)$ & 0.27 \\
\hline Invasive mechanical ventilation, $n, \%$ & $20(45.4)$ & $9(25)$ & 0.06 \\
\hline Duration of ventilator support, days $(M \pm S D)$ & $5.56 \pm 1.07$ & $5.76 \pm 0.97$ & 0.26 \\
\hline \multicolumn{4}{|c|}{ Concomitant therapy } \\
\hline Antibiotic therapy, $n, \%$ & $44(100)$ & $36(100)$ & 0.99 \\
\hline Glucocorticoid therapy, $n, \%$ & $11(25)$ & $9(25)$ & 0.99 \\
\hline Renal-replacement therapy, $n, \%$ & $4(9.1)$ & $4(11.1)$ & 0.76 \\
\hline Vasopressors, $n, \%$ & $12(27.2)$ & $6(16.6)$ & 0.26 \\
\hline
\end{tabular}

times compared to the initial values, while standard therapy did not affect these indicators significantly (Table 2).

Moreover, in comparison to standard therapy, Thymalin halved hospital mortality, increased the percentage of clinical improvement and recovery rate of the blood lymphocyte count (Table 3).

Impairment of cell-mediated adaptive immunity is quite common in COVID-19 patients; most critical cases manifest severe lymphocytopenia [1]. In a recent retrospective study, Thymosin $\alpha$ l supplement contributed to a significant reduction in the mortality rate of severe COVID-19 patients. Thymosin $\alpha 1$ effectively augmented the number of $\mathrm{CD}^{+}$or $\mathrm{CD}^{+}{ }^{+} \mathrm{T}$-cells in the older COVID-19 patients with severe lymphocytopenia [28]. Lymphopenia is a frequently reported feature of COVID-19-induced "cytokine storm" [25, 37]. Lymphopenia and leukocytosis may play a crucial role in the diagnosis and prediction of severe COVID-19 outcomes [10]. A faster reversal of lymphopenia was observed in the Thymalin group, with a remarkable recovery in the blood levels of platelets/lymphocytes ratio, $\mathrm{CD}^{+}, \mathrm{CD}^{+}{ }^{+} \mathrm{HLA}-\mathrm{DR}{ }^{+}$, B- and NK-cells subpopulations. These data are similar to the results obtained during the administration of the thymic hormone Thymosin $\alpha$, which indicates the prospects for the use of immunocorrection in severe coronavirus infection. Moreover, normalization of the immune status indicators in the older patients with severe COVID-19 against the background of Thymalin administration correlates with its immunoprotective and geroprotective properties described previously [32].

Since in most cases older persons have age-related involution of thymus [34], they are most susceptible to the development of immunopathology under bacterial and viral infections. Thymalin normalizes thymus functions during ageing process and has no side effects when used in older age groups, which is essential for the prevention of the cytokine storm in COVID-19. Based on the study conducted, Thymalin can be 


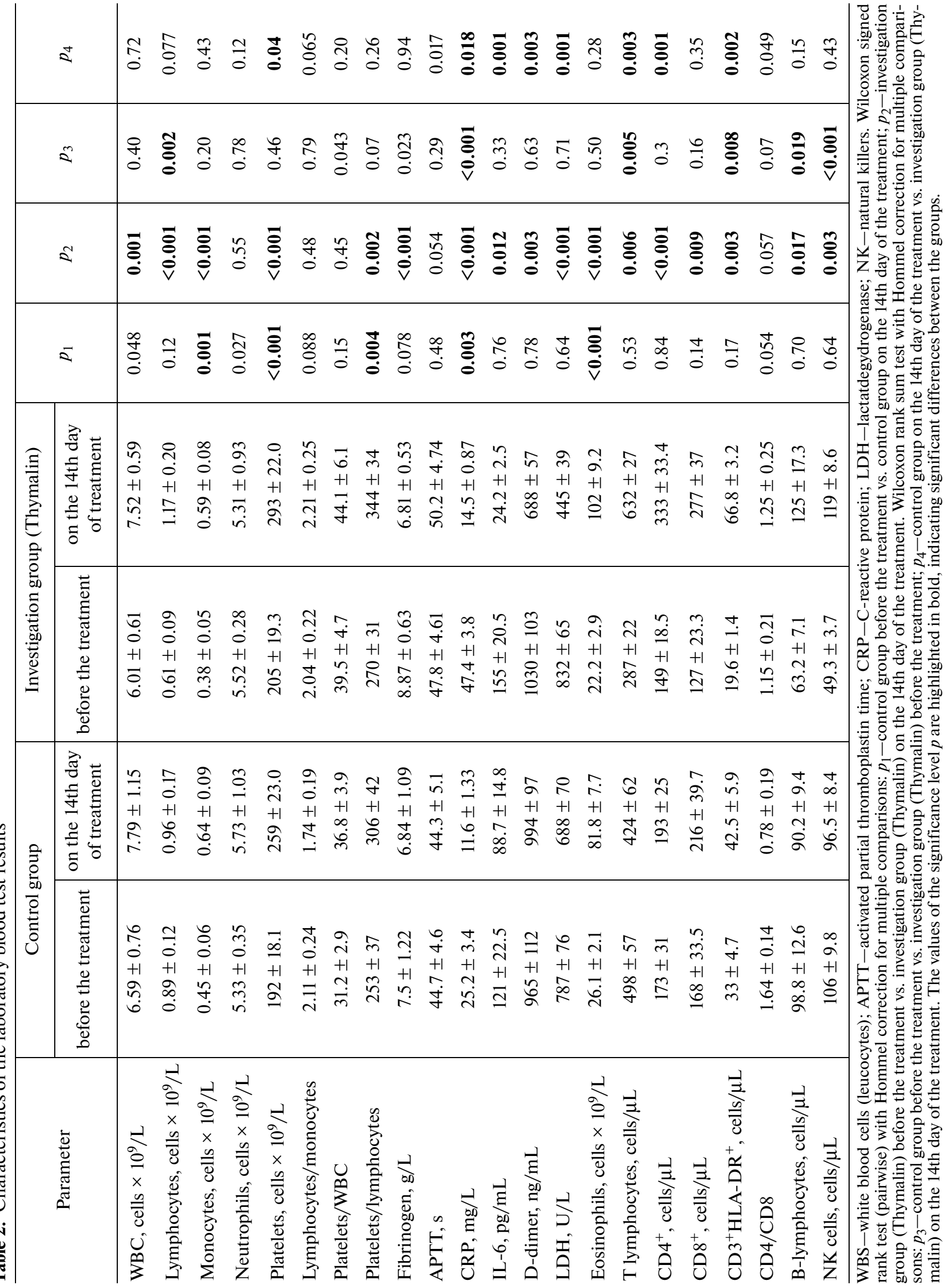


Table 3. Clinical outcome

\begin{tabular}{|c|c|c|c|}
\hline Characteristics & Control group & Investigation group (Thymalin) & $p$ \\
\hline Clinical improvement, $n, \%$ & $26(59)$ & $29(80.5)$ & $\mathbf{0 . 0 3 9}$ \\
\hline Time to clinical improvement, days $(M \pm S D)$ & $7.61 \pm 0.93$ & $8.63 \pm 0.68$ & 0.41 \\
\hline \multicolumn{4}{|c|}{ CT scan follow-up at day 14} \\
\hline Improvement, $n, \%$ & $10(22.7)$ & $11(30.5)$ & 0.43 \\
\hline Stable, $n, \%$ & $17(38.6)$ & $16(44.4)$ & 0.61 \\
\hline Progression, $n, \%$ & $17(38.6)$ & $9(25)$ & 0.19 \\
\hline \multicolumn{4}{|c|}{ Mortality } \\
\hline In hospital mortality, $n, \%$ & $18(40.9)$ & $7(19.4)$ & $\mathbf{0 . 0 3 9}$ \\
\hline Time from randomization to death, days $(M \pm S D)$ & $7.22 \pm 0.65$ & $11.2 \pm 0.49$ & 0.005 \\
\hline \multicolumn{4}{|c|}{ Laboratory recovery } \\
\hline Lymphocyte recovery, $n, \%$ & $19(43.18)$ & $24(66.6)$ & $\mathbf{0 . 0 3 7}$ \\
\hline Time to lymphocyte recovery, days $(M \pm S D)$ & $10.78 \pm 0.53$ & $7.36 \pm 0.56$ & 0.002 \\
\hline COVID-19 clearance time among survivors, days $(M \pm S D)$ & $18.27 \pm 0.38$ & $19.75 \pm 0.47$ & 0.31 \\
\hline
\end{tabular}

The values of the significance level $p$ are highlighted in bold, indicating significant differences between the groups.

assumed to improve treatment results in older patients with COVID-19, due to the above properties.

It is noteworthy, that the patients manifested the symptoms of progressive respiratory failure and the onset of the "cytokine storm", as evidenced by the increased levels of acute-phase markers (D-dimer, $C$-reactive protein, lactatdegydrogenase, IL-6), low platelets, decreased fibrinogen, lymphopenia, coagulopathy and lung tissue damage. Hyperexpression of IL-6 is a key feature of pneumonia progression in COVID-19, leading to the development of acute respiratory distress syndrome and "cytokine storm." In this regard, inhibition of IL-6 receptors may be one of the promising strategies for COVID-19 therapy [39]. During the study, normalization of laboratory abnormalities, indicative of coagulopathy (fibrinogen, APTT, D-dimer), and IL-6 blood level was observed in the Thymalin group. In addition, Thymalin halved hospital mortality in older patients with severe COVID-19. Observational and pathological studies in patients with COVID-19 have linked the cytokine storm with a pro-coagulant thrombotic milieu [29, 33]. Previous studies showed a beneficial effect of Thymalin on blood coagulation and vasomotor functions, especially when it was co-administered with heparin $[23,38]$. Since early stages of COVID-19 are associated with coagulopathy, disseminated intravascular coagulation and thrombotic angiopathy, it can be assumed that Thymalin can contribute to the effectiveness of anticoagulation therapy and prevent thrombosis in patients with severe COVID-19.

Usage of mesenchymal stem cells (MSC) is one of the newest proposed treatment strategies for severe COVID-19 [31]. Mesenchymal stem cells in patients with coronavirus infection, including severe cases, have been established to arrest the "cytokine storm," normalize the concentration of D-dimer and C-reactive protein, increase the number of $\mathrm{CD}^{+}, \mathrm{CD}^{+}$, $\mathrm{CD}^{+}$T-lymphocytes [4, 9, 22, 30]. All the listed pathogenetic components of COVID-19 are regulated by Thymalin. Thymalin, with EW, KE, EDP peptides in its composition, is a regulator of stem cell differentiation towards $T$-lymphocytes [18, 20]. This allows us to consider Thymalin as a more secure, clinically used alternative to MSC transplantation for the treatment of severe COVID-19.

Based on the analysis of the literature data on the COVID-19 pathogenesis, therapy of this disease and Thymalin biological activity, the following scheme can be proposed to substantiate its effectiveness in severe coronavirus infection (Fig. 1).

\section{CONCLUSIONS}

Short peptides EW, KE, EDP in Thymalin composition regulate the functions of stem and lymphoid cells: promote deherochromatinization, interact with histones and change the transcription availability of gene promoters, modulate gene expression and DNA methylation status. Thymalin and its constituent short peptides induce differentiation of hematopoietic stem cells into T-lymphocytes. This can contribute to normalization of the T-cell immunity function and prevent the development of a "cytokine storm." Moreover, Thymalin and peptides EW, KE, EDP normalize the hemostasis system, the deregulation of which is a key link in the pathogenesis of COVID-19. Thus, 


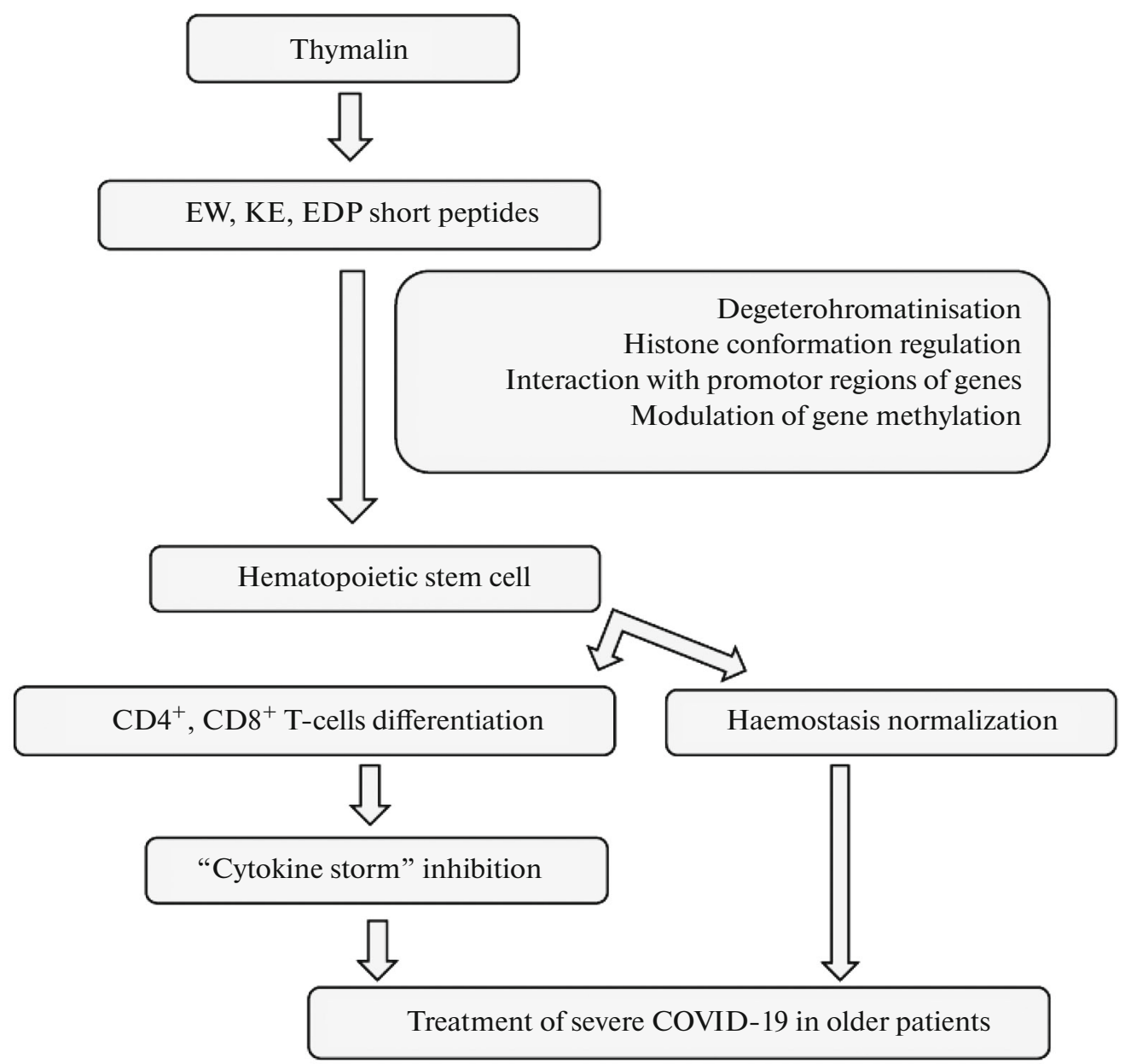

Fig. 1. The proposed mechanism of the immune status and hemostasis normalization in the treatment of COVID-19 with Thymalin.

Timalin can be considered as an effective agent for the complex therapy of COVID-19 patients.

Authors declare no conflict of interests.

\section{COMPLIANCE WITH ETHICAL STANDARDS}

Conflict of interest. The authors declare that they have no conflicts of interest.

Statement of compliance with standards of research involving humans as subjects. The trial was approved by the institutional review board of the Affiliated Hospital of Chita State Medical Academy (protocol no. 102 dated May 15, 2020) in accordance with the Declaration of Helsinki. All enrolled participants or their legal guardians have provided their written informed consent.

\section{REFERENCES}

1. Darazam, I.A., Hatami, F., Rabiei, M.M., et al., An investigation into the beneficial effects of high-dose interferon beta 1-a, compared to low-dose interferon beta 1-a (the base therapeutic regimen) in moderate to severe COVID-19: a structured summary of a study protocol for a randomized controlled 1 trial, Trials, 2020, vol. 21 , no. 1 , p. 880 . http://dx.doi.org/88010.1186/s13063-020-04812-2

2. Anisimov, V.N. and Khavinson, V.Kh., Peptide bioregulation of aging: results and prospects, Biogerontology, 2010, vol. 11, no. 2, pp. 139-149. https://doi.org/10.1007/s10522-009-9249

3. Ashapkin, V., Khavinson, V., Shilovsky, G., et al., Gene expression in human mesenchymal stem cell aging cultures: modulation by short peptides, Mol. Biol. Rep., 2020, vol. 47, no. 6, pp. 4323-4329. https://doi.org/10.1007/s11033-020-05506-3

4. Atluri, S., Manchikanti, L., and Hirsch, J.A., Expanded umbilical cord mesenchymal stem cells (UC-MSCs) as a therapeutic strategy in managing critically ill COVID-19 patients: the case for compassionate use, Pain Physician, 2020, vol. 23, no. 2, pp. E71-E83.

5. Cao, B., Wang, Y., Wen, D., et al., A trial of lopinavirritonavir in adults hospitalized with severe COVID-19, 
N. Engl. J. Med., 2020, vol. 382, no. 19, pp. 1787-1799. https://doi.org/10.1056/NEJMoa2001282

6. Fedoreyeva, L.I., Kireev, I.I., Khavinson, V.Kh., and Banyushin, B.F., Penetration of short fluorescence labeled peptides into the nucleus in HeLa cells and in vitro specific interaction of the peptides with deoxyribooligonucleotides and DNA, Biochemistry (Moscow), 2011, vol. 76, no. 11, pp. 1210-1219. https://doi.org/10.1134/s0006297911110022

7. Fedoreeva, L.I., Smirnova, T.A., Kolomijtseva, G.Ya., et al., Interaction of short peptides with FITC-labeled wheat histones and their complexes with deoxyribooligonucleotides, Biochemistry (Moscow), 2013, vol. 78, no. 2 , pp. $166-175$.

https://doi.org/10.1134/S0006297913020053

8. Frumento, G., Zuo, J., Verma, K., et al., CD117 (c-Kit) is expressed during $\mathrm{CD}^{8+} \mathrm{T}$ cell priming and stratifies sensitivity to apoptosis according to strength of TCR engagement, Front. Immunol., 2019, vol. 10, p. 468. https://doi.org/10.3389/fimmu.2019.00468

9. Golchin, A., Seyedjafari, E., and Ardeshirylajimi, A., Mesenchymal stem cell therapy for COVID-19: present or future, Stem Cell Rev. Rep., 2020, vol. 16, no. 3, pp. $427-433$.

https://doi.org/10.1007/s12015-020-09973-w

10. Henry, B., Cheruiyot. I., Vikse, J., et al., Lymphopenia and neutrophilia at admission predicts severity and mortality in patients with COVID-19: a meta-analysis, Acta Biomed., 2020, vol. 91, no. 3, p. e2020008. https://doi.org/10.23750/abm.v91i3.10217

11. Henry, B.M., De Oliveira, M.H.S., Benoit, S., et al., Hematologic, biochemical and immune biomarker abnormalities associated with severe illness and mortality in coronavirus disease 2019 (COVID-19): a meta-analysis, Clin. Chem. Lab. Methods, 2020, vol. 58, no. 7, pp. $1021-1028$.

https://doi.org/10.1515/cclm-2020-0369

12. Khavinson, V.Kh. and Anisimov, V.N., Peptide regulation of aging: 35-year research experience, Bull. Exp. Biol. Med., 2009, vol. 148, no. 1, pp. 94-98. https://doi.org/10.1007/s10517-009-0650-8

13. Khavinson, V.Kh., Nikolsky, I.S., Nikolskaya, V.V., et al., Effect of tripeptides on lymphoid and stem cells, Bull. Exp. Biol. Med., 2011, vol. 151, no. 6, pp. 722-725. http://doi.org/10.1007/s10517-011-1425-6

14. Khavinson, V.K., Kuznik, B.I., and Ryzhak, G.A., Peptide bioregulators: a new class of geroprotectors. Message 1: Results of experimental studies, Adv. Gerontol., 2013, vol. 3, no.3, pp. 225-235.

15. Khavinson, V.K., Kuznik, B.I., and Ryzhak, G.A., Peptide bioregulators: a new class of geroprotectors, Report 2. Clinical studies results, Adv. Gerontol., 2014, vol. 4, no. 4, pp. 346-361.

16. Khavinson, V.Kh., Linkova, N.S., and Tarnovskaya, S.I., Short peptides regulate gene expression, Bull. Exp. Biol. Med., 2016, vol. 162, no. 2, pp. 288-292. https://doi.org/10.1007/s10517-016-3596-7

17. Khavinson, V., Linkova, N., Diatlova, A., and Trofimova, S., Peptide regulation of cell differentiation, Stem Cell Rev. Rep., 2020, vol. 16, pp. 118-125. https://doi.org/10.1007/s12015-019-09938-8
18. Khavinson, V., Linkova, N., Dyatlova, A., et al., Peptides: prospects for use in the treatment of COVID-19, Molecules, 2020, vol. 25, no. 10, p. 4389.

https://doi.org/10.3390/molecules25194389

19. Khavinson, V.K., Linkova, N.S., Kvetnoy, I.M., et al., Thymalin: activation of differentiation of human hematopoietic stem cells, Bull. Exp. Biol. Med., 2020, vol. 170 , no. 1 , pp. $118-122$.

https://doi.org/10.1007/s10517-020-05016-z

20. Khlystova, Z.S., Kalinina, I.I., and Shmeleva, S.P., Thymalin in developing respiratory organs of human fetus, Bull. Exp. Biol. Med., 2003, vol. 135, no. 6, pp. $600-602$.

https://doi.org/10.1023/a:1025449923475

21. Kolchina, N., Khavinson, V., Linkova, N., et al., Systematic search for structural motifs of peptide binding to double-stranded DNA, Nucleic Acids Res., 2019, vol. 47 , no. 20 , pp. $10553-10563$.

https://doi.org/10.1093/nar/gkz850

22. Kuznik, B.I., Budazhapova, D.Ts., Zagrebina, L.A., et al., Effect of thymalin on blood coagulation and fibrinolysis, Farmakol. Toksikol., 1981, vol. 44, no. 4, pp. 422-425.

23. Kuznik, B.I., Vitkovskii, Yu.A., and Budazhabon, G.B., Effects of thymalin on blood coagulation and contents of proinflammatory and anti-inflammatory cytokines in patients with burns, Vestn. Khir. im. I.I. Grekova, 2000, vol. 159, no. 5, pp. 39-43.

24. Li, X., Zeng, W., Li, X., et al., CT imaging changes of corona virus disease 2019 (COVID-19): a multi-center study in Southwest China, Transl. Med., 2020, vol. 18, no. 1, p. 154. https://doi.org/10.1186/s12967-020-02324-w

25. Liu, Y., Pan, Y., Hu, Z., et al., Thymosin alpha 1 reduces the mortality of severe COVID-19 by restoration of lymphocytopenia and reversion of exhausted $\mathrm{T}$ cells, Clin. Infect. Dis., 2020, vol. 71, no. 16, pp. 2150-2157. https://doi.org/10.1093/cid/ciaa630

26. Morath, I., Hartmann, T.N., and Orian-Rousseau, V., CD44: more than a mere stem cell marker, Int. J. Biochem. Cell. Biol., 2016, vol. 81, pp. 166-173. https://doi.org/10.1016/j.biocel.2016.09.009

27. Myburgh, R., Kiefer, J.D., Russkamp, N.F., et al., Anti-human CD117 CAR T-cells efficiently eliminate healthy and malignant CD117-expressing hematopoietic cells, Leukemia, 2020, vol. 34, no. 10, pp. 26882703.

https://doi.org/10.1038/s41375-020-0818-9

28. Pedersen, S.F. and Ho, Y.C., SARS-CoV-2: a storm is raging, J. Clin. Invest., 2020, vol. 130, no. 5, pp. 22022205.

https://doi.org/10.1172/JCI137647

29. Rizk, J.G., Kalantar-Zadeh, K., Mehra, M.R., et al., Pharmaco-immunomodulatory therapy in COVID-19, Drugs, 2020, vol. 80, no. 13, pp. 1267-1292. https://doi.org/10.1007/s40265-020-01367-z

30. Sengupta, V., Sengupta, S., Lazo, A., et al., Exosomes derived from bone marrow mesenchymal stem cells as treatment for severe COVID-19, Stem Cells Dev., 2020, vol. 29 , no. 12 , pp. $747-754$.

https://doi.org/10.1089/scd.2020.0080 
31. Shu, L., Niu, C., Li, R., et al., Treatment of severe COVID-19 with human umbilical cord mesenchymal stem cells, Stem Cell Res. Ther., 2020, vol. 11, no. 1, p. 361 .

https://doi.org/10.1186/s13287-020-01875-5

32. Tsybikov, M.N., Likhanov, I.D., Borshchevskii, V.S., et al., The use of thymalin in the treatment of the acute lung abscess, Khirurgiya, 2012, no. 11. pp. 19-23.

33. Tang, N., Li, D., Wang, X., and Sun, Z., Abnormal coagulation parameters are associated with poor prognosis in patients with novel coronavirus pneumonia, $J$. Thromb. Haemostasis, 2020, vol. 18, pp. 844-847. https://doi.org/10.1111/jth.14768

34. Thomas, R., Wang, W., and Su, D.M., Contributions of age-related thymic involution to immunosenescence and inflammaging, Immun. Ageing, 2020, vol. 17, no. 2. https://doi.org/10.1186/s12979-020-0173-8

35. Terekhov, A.Yu., Kormilets, D.Yu., Linkova, N.S., et al., Peptide KE in human proteome, Bull. Exp. Biol. Med., 2020, vol. 168, no. 5, pp. 631-633. https://doi.org/10.1007/b10517-020-04767-z
36. Wang, F., Hou, H., Luo, Y., et al., The laboratory tests and host immunity of COVID-19 patients with different severity of illness, JCI Insight, 2020, vol. 5, no. 10, p. e 137799. https://doi.org/10.1172/jci.insight.137799

37. Wang, J., Jiang, M., Chen, X., and Montaner, L.J., Cytokine storm and leukocyte changes in mild versus severe SARS-CoV-2 infection: review of 3939 COVID-19 patients in China and emerging pathogenesis and therapy concepts, J. Leukocyte Biol., 2020, vol. 108, no. 1, pp. $17-41$. https://doi.org/10.1002/JLB.3COVR0520-272R

38. Wang, T., Chen, R., Liu, C., et al., Attention should be paid to venous thromboembolism prophylaxis in the management of COVID-19, Lancet Haematol., 2020, vol. 7, no. 5, pp. e362-e363. https://doi.org/10.1016/S2352-3026(20)30109-5

39. Yamada, T., Wakabayashi, M., Yamaji, T., et al., Value of leukocytosis and elevated C-reactive protein in predicting severe coronavirus 2019 (COVID-19): a systematic review and meta-analysis, Clin. Chim. Acta, 2020, vol. 509, pp. 235-243.

https://doi.org/10.1016/j.cca.2020.06.008 